\title{
Hamming Index for Some Classes of Graphs with Respect to Edge- Vertex Incidence Matrix
}

\author{
Wilda Sitorus, Saib Suwilo and Mardiningsih \\ Department of Mathematics, Universitas Sumatera Utara, Medan, Indonesia
}

\begin{abstract}
Hamming distance of a two bit strings $u$ and $v$ of length $n$ is defined to be the number of positions of $u$ and $v$ with different digit. If $G$ is a simple graph on $\mathrm{n}$ vertices and $m$ edges and $B$ is an edge-vertex incidence matrix of $G$, then every edge $e$ of $G$ can be labeled using a binary digit string of length $n$ from the row of $B$ which corresponds to the edge $e$. We discuss Hamming distance of two different edges of the graph $G$. Then, we present formulae for the sum of all Hamming distances between two different edges of $G$, particularly when $G$ is a path, a cycle, and a wheel, and some composite graphs.
\end{abstract}

Keywords Simple graph, composite graph, incidence matrix, bit string, hamming distance, hamming index

All papers within this proceedings volume have been peer reviewed by the scientific committee of the Malikussaleh International Conference on Multidisciplinary Studies (MICoMS 2017).

\section{Introduction}

Let $Z_{2}=\{0,1\}$. Notice that $\left\langle Z_{2},+_{2}\right\rangle$ is a group with binary operation addition modulo 2 . A bit string $\mathrm{x}$ of length $n$ can be thought as an element of the set $\overbrace{Z_{2} \times Z_{2} \times \cdots \times Z_{2}}^{n}$. Moreover, the set $\overbrace{Z_{2} \times Z_{2} \times \cdots \times Z_{2}}^{n}$ with binary operation defined by

$$
\mathrm{x} \oplus_{2} \mathrm{y}=\left(x_{1}+{ }_{2} y_{1}, x_{2}+{ }_{2} y_{2}, \ldots, x_{n}+{ }_{2} y_{n}\right)
$$

is a group. For a bit string $\mathrm{x} \in Z_{2} \times Z_{2} \times \cdots \times Z_{2}$ the weight of $x$, denoted by $w t(\mathrm{x})$, is the number of 1 contained in $\mathrm{x}$. For two bit strings $\mathrm{x}, \mathrm{y} \in Z_{2} \times Z_{2} \times \cdots \times Z_{2}$ the Hamming distance of $x$ and $y$, denoted by $H_{d}(\mathrm{x}, \mathrm{y})$, is the number of positions in $\mathrm{x}$ and $\mathrm{y}$ with different digits. Hamming distance can also be defined by $H_{d}(\mathrm{x}, \mathrm{y})=w t\left(\mathrm{x} \oplus_{2} \mathrm{y}\right)$ (Pless, 1998).

Let $G(V, E)$ be a simple graph on $\mathrm{n}$ vertices and $\mathrm{m}$ edges with vertex set $V=\left\{v_{1}, v_{2}, \ldots, v_{n}\right\}$ and edge set $E=\left\{e_{1}, e_{2}, \ldots, e_{m}\right\}$. If the edge $e=\{u, v\}$, we say that the edge $e$ incident to vertices $u$ and $v$. Two edges $e_{i}$ and $e_{j}$ are adjacent if they have one end vertex in common.

(C) Wilda Sitorus, Saib Suwilo, Mardiningsih. Published in the Emerald Reach Proceedings Series. Published by Emerald Publishing Limited. This article is published under the Creative Commons Attribution (CC BY 4.0) licence. Anyone may reproduce, distribute, translate and create derivative works of this article (for both commercial and non-commercial purposes), subject to full attribution to the original publication and authors. The full terms of this licence may be seen at http:// creativecommons.org/licences/by/4.0/legalcode

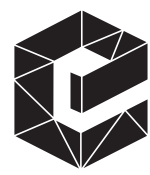


Proceedings of MICoMS 2017

An edge-vertex incidence matrix of a graph $G$ is an $m \times n$ matrix $B=\left(b_{i j}\right)$ defined by

$$
b_{i j}= \begin{cases}1, & \text { if the edge } e_{i} \text { incident to the vertex } v_{j}, \\ 0, & \text { if the edge } e_{i} \text { not incident to the vertex } v_{j} .\end{cases}
$$

Notice that every row of the edge-vertex incidence matrix of $G$ is a bit string of length $n$ and thus the edge-vertex incidence matrix contains $m$ bit strings each of length $n$. For $i=1,2, \ldots, m$ we define $s\left(e_{i}\right)=B(i,:)$ where $B(i,:)$ is the $i$ th row of $B$. For each two different edges $e_{i}$ and $e_{j}$ the Hamming distance between $e_{i}$ and $e_{j}$ is defined to be

$$
H_{d}\left(s\left(e_{i}\right), s\left(e_{j}\right)\right)=H_{d}(B(i,:), B(j,:))=w t\left(B(i,:) \oplus_{2} B(j,:)\right) .
$$

The sum of Hamming distances of a graph $G$, denoted by $H(G)$, is defined as

$$
\begin{aligned}
H(G) & =\sum_{1 \leq i<j \leq m} H_{d}\left(s\left(e_{i}\right), s\left(e_{j}\right)\right) \\
& =\sum_{1 \leq i<j \leq m} H_{d}(B(i,:), B(j,:))=\sum_{1 \leq i<j \leq m} w t\left(B(i,:) \oplus_{2} B(j,:)\right) .
\end{aligned}
$$

The sum of Hamming distances is also called the Hamming index.

The notion of Hamming distance and sum of Hamming distances of edges of simple graph $G$ is initiated by Ramane et al. (2015). They discuss a general formula for Hamming index of a graph and apply this formula to get the Hamming index of classes of regular graph. Hamming distance and Hamming index of a graph can also be defined using the adjacency matrix of the graph (Ganagi and Ramane, 2016). In this paper, we discuss Hamming distance and sum of Hamming distances of simple graph with respect to its edgevertex incidence matrix. We especially discuss a way of finding the Hamming index of a graph from the known Hamming index of its subgraph. We first present formulae for Hamming index of some graphs consist of a path or a cycle as its subgraph. We then present a formula for Hamming index of some composite graphs.

\section{Necessary background}

Let $G$ be a graph with vertex set $V=\left\{v_{1}, v_{2}, \ldots, v_{n}\right\}$ and edge set $E=\left\{e_{1}, e_{2}, \ldots, e_{m}\right\}$. For each vertex $v_{i}$ the degree of $v_{i}$, denoted by $\operatorname{deg}\left(v_{i}\right)$, is the number of edges in $G$ that are incident to $v_{i}$. It is well known that $\sum_{i=1}^{n} \operatorname{deg}\left(v_{i}\right)=2 m$ (Korte and Vygen, 2006).

We note that by definition, the Hamming index of a graph can be calculated using the formula $H(G)=\sum_{1 \leq i<j \leq m} w t\left(B(i,:) \oplus_{2} B(j,:)\right)$. Employing this formula, the Hamming index of a certain graph can be determined using the following algorithm (Figure 1).

The following lemma, due to Ramane et al. (2015), presents a formula for Hamming distance between two edges of a simple graph. We present a simpler proof than in Ramane et al. (2015).

Lemma 2. Let $G$ be a simple graph. Then

$$
H_{d}\left(s\left(e_{i}\right), s\left(e_{j}\right)\right)= \begin{cases}2, & \text { if } e_{i} \text { adjacent to } e_{j} \\ 4, & \text { if } e_{i} \text { not adjacent to } e_{j}\end{cases}
$$


Proof. Since every edge of $G$ is incident to exactly two vertices, then for each $i=1,2, \ldots, m$, we have $w t\left(s\left(e_{i}\right)\right)=2$. Let $s\left(e_{i}\right)=x_{1} x_{2} x_{3} \cdots x_{n}$ and $s\left(e_{j}\right)=y_{1} y_{2} y_{3} \cdots y_{n}$. If edge $e_{i}$ is adjacent to $e_{j}$, then there is a common vertex, say $v_{k}$, of edges $e_{i}$ and $e_{j}$. This implies $x_{k}=y_{k}=1$, and if $t \neq k$ then $x_{t}=y_{t}=0$ or $x_{t} \neq y_{t}$. Therefore, if the edge $e_{i}$ is adjacent to the edge $e_{j}$,

$$
H_{d}\left(s\left(e_{i}\right), s\left(e_{j}\right)\right)=w t\left(s\left(e_{i}\right)\right)+w\left(s\left(e_{j}\right)\right)-2=2 .
$$

If the edge $e_{i}$ is not adjacent to the edge $e_{j}$, then there is no common vertex $v_{k}$ of $e_{i}$ and $e_{j}$. This implies for each $t=1,2, \ldots, n, x_{t}=y_{t}=0$ or $x_{t} \neq y_{t}$. Therefore, if the edge $e_{i}$ is not adjacent to the edge $e_{j}$, we have

$$
H_{d}\left(s\left(e_{i}\right), s\left(e_{j}\right)\right)=w t\left(s\left(e_{i}\right)\right)+w\left(s\left(e_{j}\right)\right)=4 .
$$

Theorem 3. Let $G$ a simple graph on $m$ edges. If there are $n(P)$ adjacent pairs of edges in $G$, then $H(G)=2 m(m-1)-2 n(P)$.

Proof. By Lemma 2 we have $H(G)=2 n(P)+4 n(Q)$, where $n(Q)$ is the number of pairs of non-adjacent edges in $G$. Since the graph $G$ contains $m$ edges, then we have $n(Q)=m(m-1) / 2-n(P)$. Therefore, $H(G)=2 m(m-1)-2 n(P)$.

Proposition 4. Let $P_{n}$ a path on $n \geq 3$ vertices. Then $H\left(P_{n}\right)=2(n-2)^{2}$.

Proof. Notice that $E\left(P_{n}\right)=\left\{e_{i}=\left\{v_{i}, v_{i+1}\right\}: i=1,2, \ldots, n-1\right\}$. Therefore, there are $n(P)=n-2$ pairs of adjacent edges $e_{i}$ and $e_{i+1}$. Since $P_{n}$ has $m=n-1$ edges, then by Theorem 3 we have

$$
H\left(P_{n}\right)=2 m(m-1)-2 n(P)=2(n-1)(n-2)-2(n-2)=2(n-2)^{2} .
$$

Proposition 5. Let $C_{n}$ be a cycle on $n$ vertices. Then $H\left(C_{n}\right)=2 n(n-2)$.

Proof. There are $n$ pairs of adjacent edges $e_{i}$ and $e_{i+1}$ (we note that $e_{n+1}=e_{1}$ ), $i=1,2, \ldots, n$. Hence $n(P)=n$. By Theorem 3 we have

$$
H\left(C_{n}\right)=2 m(m-1)-2 n(P)=2 n(n-1)-2 n=2 n(n-2) .
$$

$$
\begin{aligned}
& \text { Algorithm 1: Sum of Hamming Distances } \\
& \text { Input: The edge-vertex incidence matrix B } \\
& \text { Output: Sum of Hamming distances H_B(G) } \\
& \mathrm{H} \mathrm{B}(\mathrm{G})=0 \text {; } \\
& \text { for } \mathrm{i}=1 \text { to } \mathrm{m}-1 \\
& \text { for } \mathrm{j}=\mathrm{i}+1 \text { to } \mathrm{m} \\
& \quad \mathrm{H} \_\mathrm{d}(\mathrm{ij})=w t\left(B(i,:) \oplus_{2} B(j,:)\right) \\
& \quad \mathrm{H} \mathrm{B}(\mathrm{G})=\mathrm{H} \_\mathrm{B}(\mathrm{G})+\mathrm{H} \_\mathrm{d}(\mathrm{ij}) \\
& \text { end }
\end{aligned}
$$

Figure 1.

Algorithm for Sum of Hamming Distances 


\section{Proceedings of 3. Main results}

MICoMS 2017 Let $G$ be a graph and $F$ be a subgraph of $G$. We discuss the Hamming index of the graph $G$ in term of the Hamming index of its subgraph $F$. We note that

$$
\begin{aligned}
H(G) & =\sum_{e_{i}, e_{j} \in E(G)} H_{d}\left(s\left(e_{i}\right), s\left(e_{j}\right)\right) \\
& =\sum_{e_{i}, e_{j} \in E(F)} H_{d}\left(s\left(e_{i}\right), s\left(e_{j}\right)\right)+\sum_{e_{i} \in E(F), e_{j} \notin E(F)} H_{d}\left(s\left(e_{i}\right), s\left(e_{j}\right)\right)+\sum_{e_{i}, e_{j} \notin E(F)} H_{d}\left(s\left(e_{i}\right), s\left(e_{j}\right)\right) \\
& =H(F)+\sum_{e_{i} \in E(F), e_{j} \notin E(F)} H_{d}\left(s\left(e_{i}\right), s\left(e_{j}\right)\right)+\sum_{e_{i}, e_{j} \notin E(F)} H_{d}\left(s\left(e_{i}\right), s\left(e_{j}\right)\right) .
\end{aligned}
$$

A fan $F_{1, n}$ is a graph on $n+1$ vertices and $2 n-1$ edges, where its vertex set is $V\left(F_{1, n}\right)=\left\{v_{1}, v_{2}, \ldots, v_{n+1}\right\}$ and its edge set is

$$
E\left(F_{1, n}\right)=\left\{\left\{v_{i}, v_{i+1}\right\}: i=1,2, \ldots, n-1\right\} \cup\left\{\left\{v_{i}, v_{n+1}\right\}: i=1,2, \ldots, n\right\} .
$$

Theorem 6. For a fan $F_{1, n}$, we have $H\left(F_{1, n}\right)=7 n^{2}-17 n+12$.fo

Proof. Notice that a fan $F_{1, n}$ contains the path $P_{n}$ as its subgraph where $V\left(P_{n}\right)=$ $\left\{v_{1}, v_{2}, \ldots, v_{n}\right\}$ and $E\left(P_{n}\right)=\left\{\left\{v_{i}, v_{i+1}\right\}: i=1,2, \ldots, n-1\right\}$. From Proposition 4 , we have $H\left(P_{n}\right)=2(n-2)^{2}=2 n^{2}-8 n+8$.

There are $n(n-1)$ pairs of edges $e_{i}$ and $e_{j}$ for which $e_{i} \in P_{n}$ and $e_{j} \notin P_{n}$. Among them there are $2(n-1)$ adjacent edges. This implies

$$
\sum_{e_{i} \in P_{n}, e_{j} \notin P_{n}} H_{d}\left(s\left(e_{i}\right), s\left(e_{j}\right)\right)=2(2(n-1))+4\left(n^{2}-3 n+2\right)=4 n^{2}-8 n+4 .
$$

There are $n(n-1) / 2$ pairs of edges $e_{i}$ and $e_{j}$ for which $e_{i} \notin P_{n}$ and $e_{j} \notin P_{n}$. All of them are adjacent pairs. This implies

$$
\sum_{e_{i} \notin P_{n}, e_{j} \notin P_{n}} H_{d}\left(s\left(e_{i}\right), s\left(e_{j}\right)\right)=2(n(n-1) / 2)=n^{2}-n .
$$

We now conclude that

$$
\begin{aligned}
H\left(F_{1, n}\right) & =H\left(P_{n}\right)+\sum_{e_{i} \in P_{n}, e_{j} \notin P_{n}} H_{d}\left(s\left(e_{i}\right), s\left(e_{j}\right)\right)+\sum_{e_{i}, e_{e} \notin P_{n}} H_{d}\left(s\left(e_{i}\right), s\left(e_{j}\right)\right) \\
& =\left(2 n^{2}-8 n+8\right)+\left(4 n^{2}-8 n+4\right)+\left(n^{2}-n\right)=7 n^{2}-17 n+12 .
\end{aligned}
$$

An $n$-wheel $W_{n}$ is a graph on $n+1$ vertices and $2 n$ edges with vertex set $V\left(W_{n}\right)=\left\{v_{1}, v_{2}, \ldots, v_{n+1}\right\}$ and edge set $E\left(W_{n}\right)=\left\{\left\{v_{i}, v_{i+1}\right\}: i=1,2, \ldots, n-1\right\} \cup$ $\left\{\left\{v_{n}, v_{1}\right\}\right\} \cup\left\{\left\{v_{i}, v_{i+n}\right\}: i=1,2, \ldots, n\right\}$.

Theorem 7. If $n \geq 2$, then $H\left(W_{n}\right)=7 n^{2}-9 n$.

Proof. We note that the cycle $C_{n}$ is a subgraph of $W_{n}$. Proposition 5 guarantees that $H\left(C_{n}\right)=2 n^{2}-4 n$. There are $n^{2}$ pairs of edges $e_{i}$ and $e_{j}$ for which $e_{i} \in C_{n}$ and $e_{j} \notin C_{n}$. Among them there are $2 n$ pairs of adjacent edges. This implies 


$$
\sum_{e_{i} \in C_{n}, e_{j} \notin C_{n}} H_{d}\left(s\left(e_{i}\right), s\left(e_{j}\right)\right)=2(2 n)+4\left(n^{2}-2 n\right)=4 n^{2}-4 n .
$$

Hamming Index for Some

Classes of Graphs

There are $n(n-1) / 2$ pairs of edges $e_{i}$ and $e_{j}$ for which $e_{i}, e_{j} \notin W_{n}$. All of them are adjacent pairs. This implies

$$
\sum_{e_{i}, e_{j} \notin W_{n}} H_{d}\left(s\left(e_{i}\right), s\left(e_{j}\right)\right)=2(n(n-1) / 2)=n^{2}-n .
$$

We now conclude that

$$
\begin{aligned}
H\left(W_{n}\right) & =H\left(C_{n}\right)+\sum_{e_{i} \in C_{n}, e_{j} \notin C_{n}} H_{d}\left(s\left(e_{i}\right), s\left(e_{j}\right)\right)+\sum_{e_{i}, e_{j} \notin C_{n}} H_{d}\left(s\left(e_{i}\right), s\left(e_{j}\right)\right) \\
& =\left(2 n^{2}-4 n\right)+\left(4 n^{2}-4 n+\left(n^{2}-n\right)=7 n^{2}-9 n .\right.
\end{aligned}
$$

An $n$-Sheep Steering Wheel, denoted by $S S W_{n}$, is a graph on $2 n+1$ vertices and $3 n$ edges such that its vertex set is $V\left(S S W_{n}\right)=V\left(W_{n}\right) \cup\left\{v_{n+2}, v_{n+3}, \ldots, v_{2 n+1}\right\}$ and its edge set is $E\left(S S W_{n}\right)=E\left(W_{n}\right) \cup\left\{\left\{v_{i}, v_{n+1+i}\right\}: i=1,2, \ldots, n\right\}$.

Theorem 8 . If $n \geq 3$, then $H\left(S S W_{n}\right)=17 n(n-1)$.

Proof. Notice that an $n$-wheel $W_{n}$ is a subgraph of $S S W_{n}$. If the pair of edges $e_{i}$ and $e_{j}$ are both in $W_{n}$, then by Theorem 7

$$
\sum_{e_{i}, e_{j} \in E\left(W_{n}\right)} H_{d}\left(s\left(e_{i}\right), s\left(e_{j}\right)\right)=7 n^{2}-9 n .
$$

Let the pair of edges $e_{i}$ and $e_{j}$ are such that $e_{i}$ is in $W_{n}$ and $e_{j}$ is not in $W_{n}$. There are $3 n$ adjacent pairs of such edges. There are $n(2 n-3)$ non-adjacent pairs of such edges. Therefore

$$
\sum_{e_{i} \in E\left(W_{n}\right), e_{j} \notin E\left(W_{n}\right)} H_{d}\left(s\left(e_{i}\right), s\left(e_{j}\right)\right)=2(3 n)+4(n(2 n-3))=8 n^{2}-6 n .
$$

There are $n(n-1) / 2$ pairs of non-adjacent edges $e_{i}$ and $e_{j}$ such that both of them are not in $W_{n}$. There are no pairs of adjacent edges $e_{i}$ and $e_{j}$ such that both of them are not in $W_{n}$. Therefore

$$
\sum_{e_{i}, e_{j} \notin E\left(W_{n}\right)} H_{d}\left(s\left(e_{i}\right), s\left(e_{j}\right)\right)=2 n(n-1) .
$$

We now conclude that

$$
\begin{aligned}
H\left(S S W_{n}\right) & =\sum_{e_{i}, e_{j} \in E\left(S S W_{n}\right)} H_{d}\left(s\left(e_{i}\right), s\left(e_{j}\right)\right)=7 n^{2}-9 n+8 n^{2}-6 n+2 n^{2}-2 n \\
& =17 n(n-1) .
\end{aligned}
$$


Proceedings of An $(n, m)$-Jahangir graph, denoted by $J_{n, m}$, is a graph on $n m+1$ vertices and $m(n+1)$ edges MICoMS 2017 such that its vertex set is $V\left(J_{n, m}\right)=V\left(C_{m n}\right) \cup\left\{v_{m n+1}\right\}$ and its edge set is

$$
E\left(J_{n, m}\right)=E\left(C_{m n}\right) \cup\left\{\left\{v_{(j-1) n+1}, v_{m n+1}\right\}: j=1,2, \ldots, m\right\} .
$$

Theorem 9. If $J_{n, m}$ is a $(n, m)$-Jahangir graph, then $H\left(J_{n, m}\right)=(2 n m+4 m)$ $(n m-2)+m(m+3)$.

Proof. Notice that the cycle $C_{n m}$ is a subgraph of the Jahangir graph $J_{n, m}$. If the pair of edges $e_{i}$ and $e_{j}$ are both in $C_{n m}$, then by Proposition 5 we have

$$
\sum_{e_{i}, e_{j} \in C_{n m}} H_{d}\left(s\left(e_{i}\right), s\left(e_{j}\right)\right)=H_{B}\left(C_{n m}\right)=2 n m(n m-2) .
$$

If the pair of edges $e_{i}$ and $e_{j}$ is such that $e_{i}$ is in $C_{n m}$ and $e_{j}$ is not in $C_{n m}$, then there are $2 m$ pairs of edges that are adjacent and there are $m(n m-2)$ pairs of edges which are not adjacent. Therefore

$$
\sum_{v_{i} \in V\left(C_{n n}\right), v_{j} \notin V\left(C_{n n}\right)} H_{d}\left(s\left(e_{i}\right), s\left(e_{j}\right)=2(2 m)+4 m(n m-2) .\right.
$$

If both edges $e_{i}$ and $e_{j}$ are not in $C_{n m}$, then there are $m(m-1) / 2$ pairs of edges which are adjacent and there are no pairs of edges which are not adjacent. Therefore

$$
\sum_{v_{i}, v_{j} \notin V\left(C_{n m}\right)} H_{d}\left(s\left(e_{i}\right), s\left(e_{j}\right)\right)=m(m-1) .
$$

We now conclude that

$$
\begin{aligned}
H_{B}\left(J_{n, m}\right) & =2 n m(n m-2)+4 m+4 m(n m-2)+m(m-1) \\
& =(2 n m+4 m)(n m-2)+m(m+3) .
\end{aligned}
$$

We now discuss the hamming index of two classes of composite graphs. Let $G_{1}\left(V_{1}, E_{1}\right)$ and $G_{2}\left(V_{2}, E_{2}\right)$ are two graphs. The graph $G_{1} \cup G_{2}$ is a graph with vertex set $V\left(G_{1} \cup G_{2}\right)=V\left(G_{1}\right) \cup V\left(G_{2}\right)$ and edge set $E\left(G_{1} \cup G_{2}\right)=E\left(G_{1}\right) \cup V\left(G_{2}\right)$.

Theorem 10. Let $G_{1}$ be a graph on $m_{1}$ edges and $G_{2}$ be a graph on $m_{2}$ edges. If $V\left(G_{1}\right) \cap V\left(G_{2}\right)=\varnothing$, then $H\left(G_{1} \cup G_{2}\right)=H\left(G_{1}\right)+H\left(G_{2}\right)+4 m_{1} m_{2}$.

Proof. If pairs of edges $e_{i}$ and $e_{j}$ are both $G_{1}$, then

$$
\sum_{e_{i}, e_{j} \in E\left(G_{1}\right)} H_{d}\left(s\left(e_{i}\right), s\left(e_{j}\right)\right)=H\left(G_{1}\right) .
$$

If pairs of edges $e_{i}$ and $e_{j}$ are both on $G_{2}$, then

$$
\sum_{e_{i}, e_{j} \in E\left(G_{2}\right)} H_{d}\left(s\left(e_{i}\right), s\left(e_{j}\right)\right)=H\left(G_{2}\right) .
$$


If $e_{i}$ is on $G_{1}$ and $e_{j}$ is on $G_{2}$, then $e_{i}$ and $e_{j}$ are not adjacent. Since there are $m_{1} m_{2}$ pairs of such edges, by Lemma 3 we have

$$
\sum_{e_{i} \in E\left(G_{1}\right), e_{j} \in E\left(G_{2}\right)} H_{d}\left(s\left(e_{i}\right), s\left(e_{j}\right)\right)=4 m_{1} m_{2} .
$$

Hamming Index for Some

Classes of Graphs

Therefore, $H\left(G_{1} \cup G_{2}\right)=H\left(G_{1}\right)+H\left(G_{2}\right)+4 m_{1} m_{2}$.

Let $G$ be a graph on $n$ vertices $V(G)=\left\{v_{1}, v_{2}, \ldots, v_{n}\right\}$ and $m$ edges $E(G)$. Define the thorn graph $G^{+}$of $G$ to be a graph on $2 n$ vertices and $m+n$ edges such that

$$
V\left(G^{+}\right)=V(G) \cup\left\{v_{n+1}, v_{n+2}, \ldots, v_{2 n}\right\}
$$

and

$$
E\left(G^{+}\right)=E(G) \cup\left\{\left\{v_{i}, v_{i+n}\right\}: i=1,2, \ldots, n\right\} .
$$

Theorem 11. Let $G$ be a graph on $n$ vertices and $m$ edges, then $H\left(G^{+}\right)=H(G)+4 m n+2 n^{2}-2 n-4 m$.

Proof. If the pair of edges $e_{i}$ and $e_{j}$ are both lie on $E(G)$, then by definition

$$
\sum_{e_{i}, e_{j} \in E(G)} H_{d}\left(s\left(e_{i}\right), s\left(e_{j}\right)\right)=H(G) .
$$

There are $n(n-1) / 2$ pairs of edges $e_{i}$ and $e_{j}$ where both of them are not on $E(G)$. Since $e_{i}$ and $e_{j}$ are not adjacent, then

$$
\sum_{e_{i}, e_{j} \notin E(G)} H_{d}\left(s\left(e_{i}\right), s\left(e_{j}\right)\right)=2 n(n-1) .
$$

We next consider the pairs of edges $e_{i}$ and $e_{j}$ where $e_{i} \in E(G)$ and $e_{j} \notin E(G)$. If the edge $e_{j}$ is incident to some vertex $v_{k}$ then $e_{j}$ is adjacent to $\operatorname{deg}\left(v_{k}\right)$ edges in $E(G)$ and $e_{j}$ is not adjacent to $\left(m-\operatorname{deg}\left(v_{k}\right)\right)$ edges in $E(G)$. This implies there are $\sum_{k=1}^{n} \operatorname{deg}\left(v_{k}\right)=2 m$ adjacent pairs of edges and there are $\sum_{k=1}^{n}\left(m-\operatorname{deg}\left(v_{k}\right)\right)=n m-2 m$ pairs of non-adjacent edges. This implies

$$
\sum_{e_{i} \in E(G), e_{j} \notin E(G)}^{\mid} H_{d}\left(s\left(e_{i}\right), s\left(e_{j}\right)\right)=2(2 m)+4(n m-2 m)=4 n m-4 m .
$$

Thus $H\left(G^{+}\right)=H(G)+4 n m+2 n^{2}-2 n-4 m$.

For $n \geq 3$, an $n$-Sun, denoted by $S_{n}$, is a graph on $2 n$ vertices and $2 n$ edges with $V\left(S_{n}\right)=\left\{v_{1}, v_{2}, \ldots, v_{2 n}\right\}$ and $E\left(S_{n}\right)=\left\{\left\{v_{i}, v_{i+1}\right\}: i=1,2, \ldots, n, v_{n+1}=v_{1}\right\} \cup$ $\left\{\left\{v_{i}, v_{i+n}\right\}: i=1,2, \ldots, n\right\}$. 
Proceedings of Corollary 12. If $n \geq 3$, then $H\left(S_{n}\right)=2 n(4 n-5)$.

MICoMS 2017 Proof. Notice that $S_{n}=C_{n}^{+}$. Proposition 5 guarantees that $H\left(C_{n}\right)=2 n(n-2)$. Since a cycle $C_{n}$ has $m=n$ edges, by Theorem 11 we have

$$
\begin{aligned}
H\left(S_{n}\right) & =H\left(C_{n}\right)+4 m n+2 n^{2}-2 n-4 m \\
& =2 n(n-2)+4 n^{2}+2 n^{2}-2 n-4 n=2 n(4 n-5) .
\end{aligned}
$$

\section{References}

Ganagi, A.B. and Ramane, H.S. (2016). "Hamming Distance between the String Generated by Adjacency Matrix of a Graph and their Sum”. Algebra and Discrete Mathematics, Vol. 22, No. 1, pp. 82-93.

Korte, B and Vigen, J. (2006). Combinatorial Optimization Theory and Algorithms. (3rd ed.). Springer, Berlin.

Pless, V. (1998). Introduction to the Theory of Error-Correcting Codes. John Wiley and Sons, Boston.

Ramane, H.S., Joshi, V.B., Jummannaver, R.B., Manjalapur, V.V., Patil, S.C., Shindhe, S.D., Hadimani, V. S., Kyalkonda, V.K. and Baddi, B.C. (2015). "Hamming Index of a Graph Generated by and EdgeVertex Incidence Matrix". International Journal of Mathematics, Science. and Engineeringg Application, Vol. 9, No. I, pp. 93-103.

\section{Corresponding author}

Saib Suwilo can be contacted at saib@usu.ac.id 